\title{
Qualidade de Vida e Transtornos Mentais Menores dos Estudantes de Medicina do Centro Universitário de Caratinga (UNEC) - Minas Gerais
}

\section{Quality of Life and Minor Mental Disorders of the Medical Students at the University Center of Caratinga (UNEC) - Minas Gerais}

Railly Crisóstomo Silva ${ }^{I}$ iD Alexandre de Araújo Pereira iD Eliane Perlatto Moura iD

\author{
PALAVRAS-CHAVE \\ - Qualidade de Vida. \\ - Estudantes de Medicina. \\ - Saúde Mental. \\ - Educação Médica.
}

Introdução: A qualidade de vida dos estudantes de Medicina há muito tempo tem sido objeto de atenção e pesquisas. Os fatores considerados estressores inerentes aos processos formativos na educação médica são potenciais desencadeadores de transtornos mentais com impacto na qualidade de vida. Conhecer a qualidade de vida, a saúde mental geral dos estudantes de Medicina e os fatores associados é fundamental para subsidiar ações que visem a melhorias e aperfeiçoamentos do aprendizado desses discentes. O objetivo deste estudo foi avaliar a qualidade de vida dos estudantes de Medicina do Centro Universitário de Caratinga (UNEC), localizado em Minas Gerais, a prevalência de transtornos mentais menores (TMM) e os fatores associados. Método: Trata-se de um estudo descritivo, transversal e quantitativo que foi conduzido por meio de um questionário autorrespondido contendo questões sobre dados sociodemográficos, qualidade de vida (WHOQOL-abreviado) e saúde mental geral (QSG-60 de Goldberg). Participaram do estudo 94 estudantes do curso de Medicina do UNEC, divididos em três grupos: (G1) 32 alunos do primeiro ano, (G2) 30 do terceiro ano e (G3) 32 do quinto ano. Todos preencheram o TCLE. Realizaram-se análises estatísticas descritivas de frequência, análise inferencial e análise de conglomerado. Resultados: Os estudantes percebem sua qualidade de vida como boa. O domínio de relações sociais obteve o maior escore, e o psicológico alcançou o menor escore. Não houve diferença significativa na qualidade de vida e saúde mental geral, nos diferentes períodos do curso. Observou-se correlação significativa entre a saúde mental geral com os domínios de qualidade de vida, mostrando que um pior estado da saúde mental geral vem acompanhado de uma pior qualidade de vida. A prevalência de TMM detectados na amostra analisada foi de 41,5\% entre os estudantes de Medicina do UNEC, com escores mais acentuados no domínio estresse psíquico e os menores no domínio desejo de morte. Identificou-se a presença de três grupos com perfis distintos de qualidade de vida: pior percepção da qualidade de vida (25,5\% dos estudantes), intermediária (30,9\%) e melhor (43,6\%). Conclusões: Os estudantes de Medicina pesquisados têm uma boa percepção de sua qualidade de vida. A saúde mental dos estudantes tem influência na qualidade de vida. Os resultados apontam para a necessidade de estudos qualitativos para aprofundar as informações sobre a qualidade de vida dos estudantes do UNEC. 


\section{KEYWORDS}

- Quality of Life.

- Medical Students.

- Mental Health.

- Medical Education.

Recebido em 27/10/19

Aceito em 2/3/20

\section{INTRODUÇÃO}

A qualidade de vida é considerada como a percepção do indivíduo de sua posição na vida, no contexto da cultura, nos sistemas de valores em que está inserido e em relação aos objetivos, às expectativas, aos padrões e às preocupações que o envolvem ${ }^{1}$. As abordagens gerais ou holísticas baseiam-se na premissa de que o conceito de qualidade de vida é multidimensional, apresenta uma organização complexa e dinâmica dos seus componentes e difere de pessoa para pessoa de acordo com seu contexto, e mesmo entre duas pessoas inseridas em um contexto similar, que apresentam particularidades nos modos de perceber os estímulos e responder a eles ${ }^{2,3}$. Assim, a noção de um estímulo estressor está relacionada tanto com a capacidade individual de lidar com determinadas situações como com as contingências conjunturais que ocorrem em uma rede complexa de interligações ${ }^{4}$.

O curso de Medicina é considerado uma fonte de estresse que afeta em maior ou menor grau a qualidade de vida dos estudantes ${ }^{5}$. Tornarse médico é um complexo processo de aquisição de competências, resultantes da interação de fatores individuais e ambientais. Segundo Wolf $^{6}$, o curso médico não é bom para a saúde do aluno, tendo em vista o estresse inerente ao curso, a consolidação da sua identidade, a aquisição de atitudes e valores éticos, além de um estilo de vida diferente, calcado em sacrifícios que dificultam o equilíbrio entre a vida pessoal e a acadêmica. Expectativas e responsabilidades aumentam progressivamente durante o curso, agregando tensões e angústias que afligem significativamente a saúde. Alves, Tenório, Anjos e Figueroa ${ }^{7}$ mostraram que a qualidade de vida dos estudantes de Medicina sofre desgastes no domínio psicológico, durante o curso médico.

\section{ABSTRACT}

Introduction: The quality of life of medical students has long been the object of interest and research, since, due to the stress inherent to the course, this population is vulnerable to mental disorders affecting their quality of life. Knowing about the medical students' quality of life and general mental health, as well as the related factors, is critical to support actions improving the medical students' learning process. To evaluate the medical students' Quality of Life (and related factors) at the University Center of Caratinga (UNEC). Method: This descriptive, cross-sectional and quantitative study was conducted through a self-administered questionnaire containing questions on sociodemographic data, quality of life (WHOQOL) and general mental health (Goldberg's QSG) A total of 94 medical students at UNEC - Caratinga, Minas Gerais, Brazil were divided into three groups, namely, G1 ( $1^{\text {st }}$ year), with 32 students, G2 ( $3^{\text {rd }}$ year), with 30 students, and G3 (5 $5^{\text {th }}$ year), with 32 students, who were analyzed through descriptive analysis, with the use of tools such as Chi-square test, Pearson's correlation, Student's t-test andlor Analysis of Variance (ANOVA). Cluster analysis was performed at the statistical significance of a $5 \%$ probability. Results: Students perceive their quality of life as good. The domain no significant difference in quality of life and general mental health, in the different periods of the course. A significant correlation was observed between general mental health (QSG) and quality of life factors, wherein a worse state of general mental health is associated with a lower quality of life. The incidence of minor mental disorders detected in the sample under analysis was of $41.5 \%$ among the students, of which the highest-scoring disorder was psychic stress (64.1\%), and the lowest one death wish (39.8\%). Conclusion: The analyzed medical students have a good perception of their quality of life. The course level (years at medical school) had no significant influence. The minor mental disorders in the population under study influences the quality of life. The results indicate the need for qualitative studies to acquire more information about the QOL of UNEC students. 
do primeiro ano, 30 do terceiro e 32 do quinto. A amostra foi determinada por conveniência. Os critérios de inclusão no estudo foram: os estudantes deveriam estar regularmente matriculados e cursar o primeiro, terceiro e quinto anos (pois representam o início, a metade e a fase final) do curso de Medicina do UNEC, ter mais de 18 anos e ter assinado o Termo de Consentimento Livre e Esclarecido (TCLE).

Os questionários foram aplicados na própria instituição, em horário pré-agendado com os alunos. Durante o encontro, os alunos foram informados sobre os objetivos da pesquisa, o modo de aplicação e o destino dos dados obtidos, seus eventuais riscos e benefícios, sua importância no desenvolvimento de métodos e atitudes que visem proporcionar mais qualidade de vida aos estudantes, bem como a possibilidade de retirada do consentimento em qualquer etapa da pesquisa.

No estudo, adotaram-se os seguintes instrumentos: um questionário para a avaliação do perfil sociodemográfico (nove questões), o WHOQOLabreviado (26 questões) voltado à qualidade de vida e o Questionário de Saúde Geral de Goldberg (QSG) (60 questões).

O questionário sociodemográfico constou de nove questões para identificação dos fatores, pessoais, familiares e do ambiente de ensino: sexo, idade, estado civil, procedência, atividade remunerada, renda familiar, tempo gasto para chegar à faculdade e motivação para fazer o curso.

Para a avaliação da qualidade de vida, foi utilizada a versão brasileira do questionário WHOQOL-abreviado, baseado na versão abreviada do Instrumento de Qualidade de Vida da Organização Mundial de Saúde (OMS). Trata-se de um instrumento muito utilizado em pesquisas nacionais e internacionais com estudantes de Medicina que mede a qualidade de vida geral autopercebida. Esse instrumento é composto por duas perguntas gerais que fazem referência à percepção da qualidade de vida e à satisfação com a saúde. As demais representam as 24 facetas que compõem o instrumento original e estão distribuídas em quatro domínios: físico, psicológico, relações sociais e meio ambiente. Os domínios e suas respectivas facetas apresentam aspectos objetivos e subjetivos para a avaliação, e as respostas são dadas em uma escala do tipo Likert. As respostas da escala variam de intensidade (nada - extremamente), capacidade (nada - completamente), frequência (nunca - sempre) e avaliação (muito insatisfeito - muito satisfeito e muito ruim - muito bom $)^{12,13}$. O cálculo dos escores de avaliação da qualidade de vida foi feito separadamente em cada um dos quatro domínios. A pontuação bruta foi transformada para uma escala de 0 a 100 (escore transformado ET 0-100) de acordo com syntax para SPSS, proposta pela OMS. Assim, o valor mínimo dos escores de cada domínio é 0 e o máximo é 100, de forma que quanto maior o escore, mais positiva é a avaliação do domínio ${ }^{12,13}$.

Para a avaliação da saúde mental dos estudantes, utilizou-se o Questionário de Saúde Geral (QSG), originalmente elaborada por Goldberg $^{14}$ e validada para o Brasil por Pasquali, Miranda e Ramos ${ }^{15}$. Essa escala visa avaliar a saúde mental das pessoas por meio de itens que indicam a presença ou ausência de sintomas ou distúrbios clínicos não psicóticos ou TMM. O QSG permite identificar pessoas com perfil sintomático (com escores acima do percentil 90), casos limítrofes (entre 85 e 90) e pessoas sem perfil sintomático (abaixo de 85). Trata-se de uma escala multifatorial que contém cinco subescalas: 1 . estresse psíquico; 2 . desejo de morte; 3 . desconfiança do próprio desempenho; 4. distúrbios do sono; 5. distúrbios psicossomáticos. Fornece ainda um escore global que indica a gravidade dos problemas de saúde mental. A escala possui
60 itens, com alternativas de respostas em escala do tipo Likert de quatro pontos, variando de "menos do que de costume" (ponto 1) a "muito mais que de costume" (ponto 4), quando o item é formulado como sintoma e o sentido inverso, quando o item expressa comportamento normal. Por meio do QSG, mediu-se a saúde mental geral dos estudantes de Medicina considerando a média de escores obtidos nos diferentes domínios da escala. A média geral do questionário superior a 2,33 foi considerada acima do percentil 90, que indica distúrbio mental ${ }^{15}$. No QSG, quanto maior for o escore (que pode variar de 1 a 4), pior será o estado de saúde mental.

Para a análise dos dados, realizou-se estatística descritiva e conduziuse uma análise univariada, em que se utilizaram o teste $t$ de Student $\mathrm{e}$ a Análise de Variância (ANOVA) com o objetivo de realizar uma préseleção das variáveis de interesse para a condução da análise multivariada, em que foi aplicada a análise de Regressão Linear Múltipla. O nível de significância utilizado foi de $5 \%(\mathrm{p}<0,05)$. Utilizou-se o programa SPSS 14.0 for Windows (Software Estatístico).

O projeto foi encaminhado ao Comitê de Ética em Pesquisa da Universidade José do Rosário Vellano e recebeu parecer favorável: Certificado de Apresentação para Apreciação Ética (CAAE) $n^{\circ}$ 88050718.4.0000.5143.

\section{RESULTADOS}

\section{Perfil sociodemográfico}

O curso de Medicina do UNEC continha 172 alunos matriculados regularmente nos anos em que se propôs o estudo (50 alunos matriculados no primeiro ano, 60 alunos no terceiro e 62 alunos no quinto). Participaram do estudo 94 alunos de Medicina, representando $49,4 \%$ do total de estudantes matriculados nos períodos analisados (64\% do primeiro ano, $50 \%$ do terceiro e $51,1 \%$ do quinto). Na amostra estudada, $58,5 \%$ eram do sexo feminino e $41,5 \%$ do sexo masculino. Em relação à faixa etária: 4,3\% tinham entre 15 e 18 anos; 58,5\%, entre 19 e 24 anos; $15,9 \%$, entre 25 e 30 anos; e 21,3\% eram maiores de 30 anos. Quanto ao estado civil, $80,8 \%$ eram solteiros, $14,9 \%$ eram casados e $4,3 \%$ eram separados ou viúvos ou viviam uma união estável. Dos discentes, 92,5\% eram procedentes de área urbana (sendo $61,7 \%$ da cidade-sede do UNEC e 30,9\% de outro estado) e $7,4 \%$ de área rural. Aproximadamente $87 \%$ dos alunos não exerciam qualquer atividade remunerada, e a renda familiar para a maioria $(71,3 \%)$ era superior a cinco mil reais. Os resultados mostraram ainda que $47,9 \%$ dos alunos residiam com a família, $23,4 \%$ moravam sozinhos e $22,3 \%$ residiam em república. O tempo gasto de casa até o UNEC era de 15 a 30 minutos para 93,6\% dos alunos. Ressalta-se que a medicina era a profissão desejada para $85,1 \%$ dos alunos e $14,9 \%$ estavam fazendo o curso por influência de familiares/outros.

\section{Análise do questionário de Qualidade de Vida (WHOQOL-abreviado)}

Para a amostra total de estudantes avaliados, a média geral ( \pm desvio padrão) da qualidade de vida foi de $63 \pm 16,5$. A análise dos escores da qualidade de vida dos estudantes de Medicina nos diferentes domínios avaliados pelo instrumento demonstrou domínios médios, em ordem decrescente, de 64,3 \pm 19,6 para o domínio das relações sociais, $64,2 \pm 15,8$ para o domínio físico, 63,0 $\pm 13,6$ para o domínio meio ambiente e o menor escore foi $60,5 \pm 17$ para o domínio psicológico (Tabela 1). Não foi observada diferença significativa nos escores médios dos estudantes nos domínios abordados no questionário de qualidade de vida, nos diferentes momentos do curso (início, meio e final).

REVISTA BRASILEIRA DE EDUCAÇ̃̃o MÉDICA

3 $44(2)$ : e064; 2020 
Quando se avaliaram de forma individual os itens que compõem cada domínio, observou-se que, no domínio relações sociais, o item mais bem avaliado foi o acesso às informações necessárias no dia a dia, ao passo que a satisfação com a vida sexual e com o ambiente físico foi o item menos pontuado. No domínio físico, o item que recebeu maior pontuação foi a capacidade de locomoção e o que recebeu a menor pontuação foi o item relacionado com a satisfação com o sono. A análise do domínio meio ambiente demonstrou que os estudantes estão muito satisfeitos com as condições do local onde moram e com o meio de transporte, entretanto o item relacionado com oportunidades de atividades de lazer foi o que recebeu a menor pontuação. No domínio psicológico, o que chamou a atenção foi a média relativamente alta $(3,1 \pm 1,1)$ no item que avalia a frequência de sentimentos negativos (mau humor, desespero, ansiedade e depressão).

Neste estudo, pesquisou-se a influência de variáveis sociodemográficas dos estudantes de Medicina do UNEC, a saber: sexo, idade, estado civil, procedência, exercer atividade remunerada, renda familiar, com quem reside (familiares, amigos, outros), tempo gasto para chegar à universidade, opção pelo curso (ser desejada, outros), nos diferentes domínios da qualidade de vida. Das variáveis analisadas, somente o estado civil apresentou diferença significativa com o domínio relações sociais, e os estudantes solteiros apresentaram escores mais elevados.

Uma análise de conglomerados foi realizada com o objetivo de identificar a existência de grupos distintos de alunos em relação aos fatores de qualidade de vida. Identificou-se a presença de três grupos com perfis de qualidade de vida distintos: o primeiro grupo (grau I) com pior percepção da qualidade de vida era composto por $25,5 \%$ dos alunos; o segundo grupo (grau II) com percepção da qualidade de vida intermediária, por 30,9\%; e o terceiro grupo (Grau III) com melhor percepção da qualidade de vida, por $43,6 \%$ (Tabela 2 ). Não se observou influência das variáveis sociodemográficas avaliadas neste estudo nos grupos com graus de qualidade de vida distintos.

\section{Análise do Questionário de Saúde Geral (QSG)}

A prevalência global de TMM na amostra analisada foi de 41,5\%. Não houve associação do ano do curso com a prevalência de TMM. O estresse psíquico foi o problema de saúde mental que apresentou maior prevalência $(64,1 \%)$, seguido por falta de confiança na capacidade de desempenho $(57,4 \%)$, distúrbios do sono $(56,5 \%)$, distúrbios psicossomáticos $(54,9 \%)$ e desejo de morte $(38,8 \%)$. Na análise dos itens que compõem cada subescala, observou-se que, na subescala estresse psíquico, o item que recebeu o maior escore foi o sentimento de que o sono não foi suficiente para renovar as energias, seguido por sentimento de cansaço-irritação e de estar constantemente sob tensão. Na subescala falta de confiança na capacidade de desempenho, o item menos pontuado foi o relacionado com o sentimento de estar desempenhando uma função útil na vida. $\mathrm{Na}$ subescala distúrbios do sono, o item com maior destaque foi a perda de sono por causa de preocupações. Em relação à escala distúrbios psicossomáticos, destacou-se o sentimento de cansaço e exaustão.

Relação entre a percepção de qualidade de vida e a percepção de saúde mental geral

Observou-se correlação significativa, de moderada a forte, entre a saúde geral e todos os fatores avaliados no questionário de qualidade de vida, nos estudantes de Medicina do UNEC. Os resultados estão

\begin{tabular}{|c|c|c|c|c|}
\hline \multicolumn{5}{|c|}{$\begin{array}{l}\text { Valores médios ( } \pm \text { dp) da pontuação nos diferentes domínios do } \\
\text { questionário de Qualidade de Vida - Whoquol-bref, dos alunos de } \\
\text { medicina do Centro Universitário de Caratinga (UNEC) - } 2018\end{array}$} \\
\hline \multirow{2}{*}{ Domínios } & \multicolumn{4}{|c|}{ Medidas descritivas } \\
\hline & n & Mínimo & Máximo & $\bar{x} \pm d p$ \\
\hline Físico & 94 & 21,4 & 96,4 & $64,2 \pm 15,8$ \\
\hline Psicológico & 94 & 25,0 & 91,7 & $60,5 \pm 17,0$ \\
\hline Relações sociais & 94 & 8,3 & 100,0 & $64,3 \pm 19,6$ \\
\hline Meio ambiente & 94 & 21,9 & 87,5 & $63,0 \pm 13,6$ \\
\hline
\end{tabular}

Base de dados: 94 alunos.

Fonte: Dados do estudo.

\begin{tabular}{|c|c|c|c|c|c|}
\hline \multirow{2}{*}{$\begin{array}{c}\text { Escalas de qualidade } \\
\text { de vida }\end{array}$} & \multicolumn{3}{|c|}{ Grau de qualidade de vida } & \multicolumn{2}{|c|}{ ANOVA } \\
\hline & Grau I & Grau II & Grau III & $\mathbf{p}$ & Conclusão \\
\hline Domínio físico & 44,9 & 63,8 & 75,9 & $<0,001$ & $I<I I<I I I$ \\
\hline Domínio psicológico & 41,0 & 54,5 & 76,1 & $<0,001$ & $I<I I<I I I$ \\
\hline Relações sociais & 40,6 & 65,8 & 77,0 & $<0,001$ & $I<I I<I I I$ \\
\hline Meio ambiente & 50,1 & 62,6 & 70,8 & $<0,001$ & $I<I I<I I I$ \\
\hline
\end{tabular}

Base de dados: 94 alunos.

A probabilidade de significância $(\mathrm{p})$ refere-se à Análise de Variância.

Fonte:Dados do estudo.

representados no Gráfico 1 . Houve correlação entre a saúde mental dos estudantes e o perfil de qualidade de vida, e aqueles com o menor grau de qualidade de vida apresentaram a pior percepção de saúde mental (escores maiores significam pior percepção da saúde mental).

\section{DISCUSSÃO}

Os achados de qualidade de vida obtidos por meio do WHOQOLabreviado revelaram que os estudantes de medicina do UNEC percebem que a sua qualidade de vida é boa. Esses resultados corroboram os achados de Chehuen Neto, Sirimarco, Pittondo, Marques e Baratti ${ }^{16}$ e Ramos-Dias, Libardi, Zillo, Igarashi e Senger ${ }^{17}$ que, ao estudarem a qualidade de vida dos alunos de Medicina da Universidade Federal de Juiz de Fora (UFJF) e da Faculdade de Ciências Médicas e da Saúde Pública da Pontifícia Universidade Católica de São Paulo - PUC-SP (câmpus de de Sorocaba), respectivamente, também observaram que os estudantes têm uma boa percepção de sua qualidade de vida. No tocante à qualidade de vida ao longo da graduação, a literatura apresenta dados controversos. Alguns estudos relatam que a qualidade de vida dos estudantes de Medicina piora no decorrer do curso ${ }^{7,18-20}$ e outros apontam que ela não se altera ${ }^{16,21} \cdot \mathrm{Na}$ amostra desta pesquisa, não houve diferença na percepção de qualidade de vida dos estudantes, nos três momentos estudados. Segundo Bampi, Baraldi, Guilhem, Araújo e Campos ${ }^{18}$, isso ocorre porque a medicina

REVISTA BRASILEIRA DE EDUCAÇÃO MÉDICA

4 44 (2) : e064; 2020 


\section{Gráfico 1}

Correlação entre os escore obtidos no Questionário de Saúde Geral (QSG) e nos diferentes domínios do questionário de Qualidade de Vida -

Whoquol-bref, dos alunos de medicina do Centro Universitário de Caratinga (UNEC), 2018
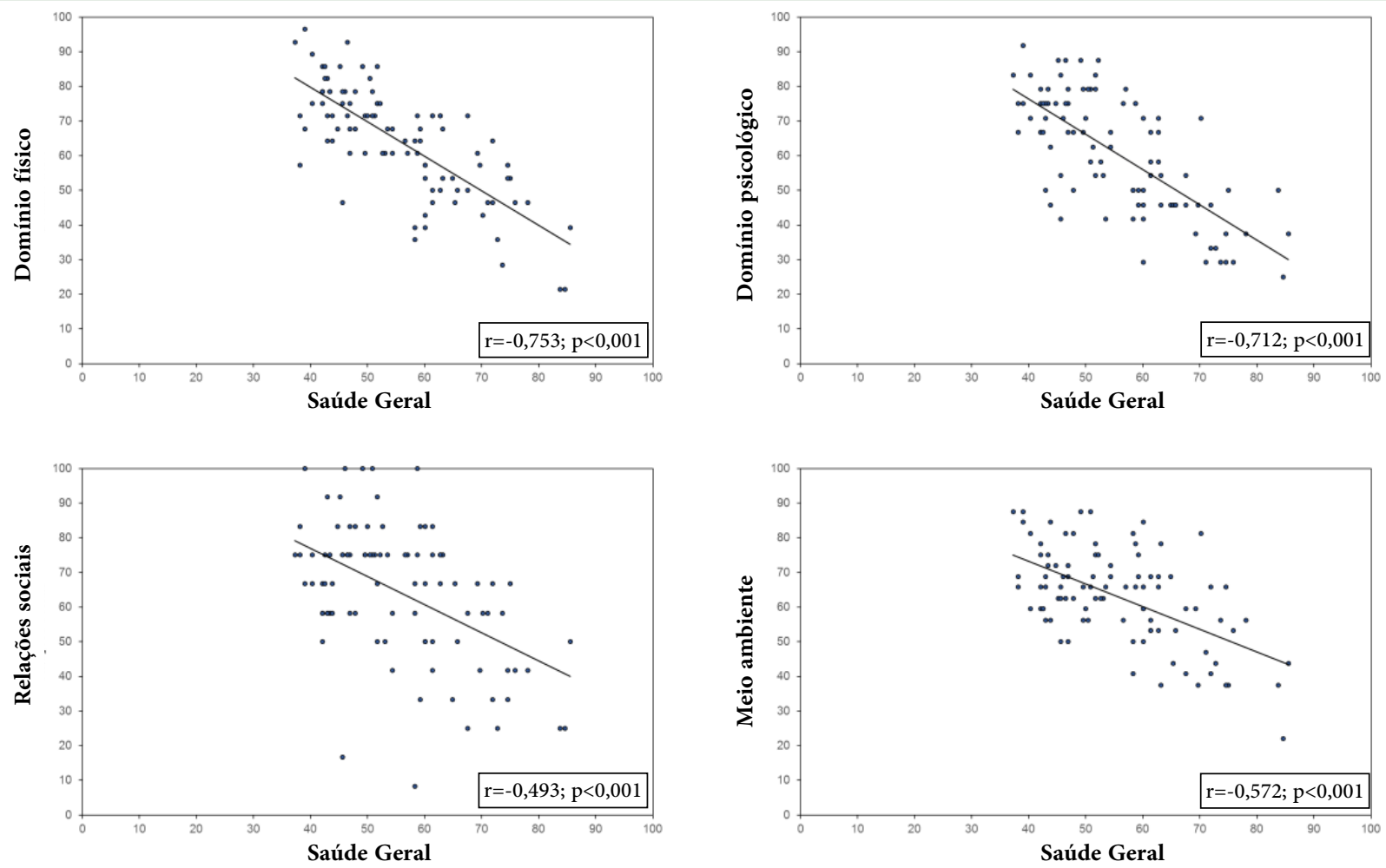

Base de dados: 94 alunos.

A probabilidade de significância $(\mathrm{p})$ refere-se à correlação de Pearson (r).

Fonte:Dados do estudo

é considerada, em termos profissionais e culturais, uma profissão de muita responsabilidade e múltiplas cobranças, o que faz com que os estudantes julguem normal lidar com o estresse gerado pelas pressões inerentes ao curso. E mesmo vivendo sob pressões e exigências do dia a dia, os estudantes geralmente mantêm, de forma equilibrada e boa, a sua qualidade de vida, sendo capazes de equacionar e superar as conhecidas e esperadas dificuldades ${ }^{16}$.

Apesar da boa percepção de qualidade de vida dos estudantes, observou-se, neste estudo, que o domínio psicológico, que avalia sentimentos positivos e negativos, memória, concentração e autoestima, apresentou menor escore. Escores mais baixos no domínio psicológico são relatados em vários estudos referentes à qualidade de estudantes de Medicina. Fieodrippe, Brandão e Valente, ao avaliar a qualidade de vida de estudantes de Medicina brasileiros oriundos de 75 escolas médicas brasileiras, públicas e privadas, observou que os piores escores foram encontrados nos domínios psicológico e de relações sociais. Bampi, Baraldi, Guilhem, Araújo e Campos ${ }^{18}$, ao avaliarem a qualidade de vida de estudantes de Medicina da Universidade de Brasília (UnB), observaram que o domínio psicológico foi o que recebeu menor escore. Alves, Tenório, Anjos e Figueroa ${ }^{7}$, ao estudarem a qualidade de vida de estudantes de Medicina da cidade do Recife, observaram desgaste no domínio psicológico ao longo do curso.

Relatos da literatura indicam que a qualidade de vida dos estudantes de Medicina é influenciada por capacidade de concentração, sono, grau de energia, capacidade para realizar atividades do dia a dia e do trabalho, oportunidades de lazer e sentimentos negativos (mau humor, desespero, ansiedade e depressão ${ }^{18}$. Neste estudo, os estudantes avaliados relataram que tinham sentimentos negativos (mau humor, desespero, ansiedade e depressão) com alta frequência, aliados à baixa qualidade de sono e ao pouco tempo para o lazer. O sono é uma função biológica essencial à manutenção da existência. Os transtornos do sono podem acarretar alterações significativas no funcionamento físico, ocupacional, cognitivo e social do indivíduo com repercussões na perda da qualidade de vida, na disfunção autonômica, na diminuição do desempenho profissional ou acadêmica e no aumento na incidência de transtornos psiquiátricos ${ }^{22,23}$. Os estudantes de Medicina compõem um grupo suscetível aos transtornos do sono, em razão da carga curricular em horário integral, das atividades extracurriculares, da forte pressão e do estresse, com exigência de alto rendimento e tempo demandado em estudos, bem como ao desenvolvimento de quadros de ansiedade $e^{24,25}$. A falta de confiança na capacidade de desempenho desencadeia estresse psíquico que pode levar a distúrbios psicossomáticos, relacionados à dificuldade dos estudantes em exteriorizar as emoções elaboradas em situações conflitantes, o que resulta em manifestações psicossomáticas que impactam a qualidade de $v \operatorname{sida}^{26}$. A saúde mental é, portanto, um fator determinante da qualidade de vida dos estudantes de Medicina ${ }^{5,21,27}$. Segundo dados da literatura, a prevalência de TMM na população brasileira varia em torno de $22 \%{ }^{28,29}$ e na população universitária está em torno de $34 \%$, e, especificamente

\footnotetext{
$5 \mid$ REVISTA BRASILEIRA DE EDUCAÇÃO MÉDICA
} 
em estudantes de Medicina, essa prevalência varia de $31,7 \%$ a $58,8 \%{ }^{8,30,31}$. Neste estudo, a prevalência de TMM nos estudantes de Medicina avaliados foi alta $(41,5 \%)$, com predomínio de estresse psíquico, seguido por falta de confiança no próprio desempenho, distúrbios do sono e distúrbios psicossomáticos. Os estudantes analisados relataram insatisfação com a qualidade do sono e sentimento de cansaço e exaustão, o que pode estar relacionado com a perda do sentimento de estar realizando algo útil na vida. Este estudo apontou ainda correlação entre a presença de TMM e a piora da qualidade de vida, em todos os domínios avaliados. Nossos resultados se mostraram semelhantes ao estudo conduzido por Soares e Moura-Faico ${ }^{32}$ com estudantes de Medicina na cidade de Caratinga, o qual encontrou prevalência de 45,3\% de TMM, identificada por meio do QSG-60 de Goldberg, e 20,5\% de desejo de morte.

Esses resultados são importantes, pois enfatizam pontos vulneráveis, indicando a necessidade de criação de estratégias educacionais que visem minimizar o impacto do curso na qualidade de vida na saúde mental dos estudantes. Zonta, Robles e Grosseman ${ }^{33}$ apontaram as seguintes estratégias para reduzir o estresse durante o curso médico: valorização dos relacionamentos interpessoais e de fenômenos do cotidiano; equilíbrio entre estudo e lazer; organização do tempo; cuidados com a saúde, a alimentação e o sono; prática de atividade física; religiosidade; trabalhar a própria personalidade para lidar com situações adversas; procura por assistência psicológica.

\section{CONSIDERAÇÕES FINAIS}

Nossos resultados demonstram que os estudantes de Medicina do UNEC, apesar do apoio psicopedagógico disponível na instituição, apresentam alto índice de TMM, com impacto na qualidade de vida.

Novos estudos são necessários para avaliar de forma mais aprofundada os fatores que impactam a qualidade de vida e a saúde mental dos estudantes de Medicina, possíveis fatores que impedem a procura por ajuda, bem como possíveis lacunas presentes no atendimento ao estudante de Medicina do UNEC.

Como limitação do estudo, pode-se citar o seu desenho. Um desenho de coorte prospectivo durante os seis anos do curso médico seria mais apropriado para acompanhar a trajetória de um mesmo grupo e conhecer as mudanças em sua qualidade de vida no decorrer da graduação. A opção por um estudo transversal se deu em função do tempo disponível para a realização da pesquisa. Este estudo foi de caráter quantitativo, sem possibilidade de investigação e checagem em profundidade acerca de questionamentos suscitados, como a compreensão sobre as percepções dos alunos sobre qualidade de vida e saúde e sobre a relação entre a sua qualidade de vida e o percurso da formação médica.

Por fim, destaca-se a importância de se provocar no meio acadêmico a reflexão de aspectos relacionados à qualidade de vida dos estudantes universitários da área da saúde, uma vez que conhecer a realidade em seu período de formação profissional pode possibilitar a criação de mecanismos de suporte para o enfrentamento das adversidades, da saúde mental geral e qualidade de vida no âmbito da própria faculdade.

\section{REFERÊNCIAS}

1. WHOQOL Group. The World Health Organization Quality of Life Assessment (WHOQOL): Position paper from the World Health Organization. Soc. sci. med. 1995;10:1403-9.

2. Farquhar M. Definitions of quality of life: a taxonomy. J. adv. nurs.
1995;22(3)502-8.

3. Pereira EF, Teixeira CS, Santos A. Qualidade de vida: abordagens, conceitos e avaliação. Rev. bras. educ. fís. esp. 2012;26(2)241-50.

4. Margis R, Picon P, Cosner AF, Silveira RO. Relação entre estressores, estresse e ansiedade. Rev. psiquiatr. Rio Gd. Sul 2003;25(Supl 1):65-74.

5. Feodrippe ALO, Brandão MCF, Valente TCO. Qualidade de vida de estudantes de Medicina: uma revisão. Rev. bras. educ. med. 2013;37(3):418-28.

6. Wolf TM. Stress, coping and health: enhancing well-being during medical school. Med. educ. 1994;28(1):8-17.

7. Alves JGB, Tenório M, Anjos, AG, Figueroa JN. Qualidade de vida em estudantes de Medicina no início e final do curso: avaliação pelo WHOQOL-bref. Rev. bras. educ. med. 2010;34(1):91-6.

8. Aragão JC, Casiraghi B, Mota É, Abrahão M, Almeida T, Baylão AC, et al. Saúde mental em estudantes de medicina. Revista de Estudios e Investigación en Psicología e Educación 2017; (14): 38-41 [acesso em 23 jul. 2019]. Disponível em: http://revistas.udc.es/index.php/reipe/ article/view/reipe.2017.0.14.2267.

9. Fiorotti KP, Rossoni RR, Borges LH, Miranda AE. Transtornos mentais comuns entre os estudantes do curso de medicina: prevalência e fatores associados. J. bras. psiquiatr. 2010;59(1):17-23.

10. Lima MCP, Domingues MS, Ramos ATAC. Prevalência e fatores de risco para transtornos mentais comuns entre estudantes de medicina. Rev Saúde Públ 2006;40(6):1035-41.

11. Goel AD, Akarte SV, Agrawal SP, Yadav V. Longitudinal assessment of depression, stress, and burnout in medical students. J Neurosci Rural Pract 2016;7:493-8.

12. Fleck MPA, Leal OF, Louzada S, Xavier M, Chachamovich E, Vieira $\mathrm{G}$, et al. Desenvolvimento da versão em português do instrumento de avaliação de qualidade de vida da OMS (WHOQOL-100). Rev. bras. psiquiatr. 1999;21(1):19-21.

13. Fleck MPA, Louzada S, Xavier M, Chachamovich E, Vieira G, Santos L, et al. Aplicação da versão em português do instrumento abreviado de avaliação de qualidade de vida WHOQOL-bref. Rev Saúde Públ 2000; 349(2):198-205.

14. Goldberg, D. The detection of psychiatric illness by questionnaire. London: Oxford University Press; 1972.

15. Pasquali, L, Miranda FJ, Ramos ALM. Questionário de Saúde Geral de Goldberg (QSG): adaptação brasileira. Psic Teor e Pesq 1994;10(3):421-37.

16. Chehuen Neto JA, Sirimarco MT, Pittondo MS, Marques FS, Baratti AB. Qualidade de vida dos estudantes de medicina e direito. HU rev. 2008;34(3):197-203.

17. Ramos-Dias JC, Libardi MC, Zillo CM, Igarashi MH, Senger MH. Qualidade de vida em cem alunos do curso de Medicina de Sorocaba PUC/SP. Rev. bras. educ. med. 2010;34(1):116-23.

18. Bampi LNS, Baraldi S, Guilhem D, Araújo MP, Campos ACO. Qualidade de vida de estudantes de Medicina da Universidade de Brasília. Rev. bras. educ. med. 2013;37(2):217-25.

19. Paro CA, Bittencour ZZLC. Qualidade de vida de graduandos da área da saúde. Rev. bras. educ. med. 2013;37(3):365-75.

20. Chazan ACS, Campos MR. Qualidade de vida de estudantes de Medicina medida pelo WHOQOL-bref - UERJ, 2010. Rev. bras. educ. med. 2013;37(3):376-84.

\footnotetext{
$6 \mid$\begin{tabular}{l|l} 
REVISTA BRASILEIRA DE EDUCAÇÃO MÉDICA \\
44 (2) : e064; 2020
\end{tabular}
} 
21. Voltmer E, Rosta J, Aasland OG, Spahn C. Study-related health and behavior patterns of medical students: a longitudinal study. Med. teach. 2010;32(10):422-8.

22. Ribeiro CR, Silva YM, Oliveira SM. O impacto da qualidade do sono na formação médica. Rev. Soc. Bras. Clín. Méd. 2014;12(1):8-14.

23. Medeiros ALD, Lima PF, Almondes KM, Dias Junior AS, Rolim SAM, Araújo JF. Hábitos de sono e desempenho em estudantes de medicina. RBCS 2002;16(1):49-54.

24. Baldassin S, Martins LC, Andrade A. Traços de ansiedade entre estudantes de medicina. Arqui Med ABC 2006;31(1):27-31.

25. Cardoso HC, Bueno FCC, Mata JC, Alves APR, Jochims I, Vaz Filho $\mathrm{IH}$, et al. Avaliação da qualidade do sono em estudantes de medicina. Rev. bras. educ. med. 2009;33(3):349-55.

26. Cerchiari EAN, Caetano D, Faccenda O. Prevalência de transtornos mentais menores em estudantes universitários. Estud. psicol. 2005;10(3):413-20.

27. Dyrby N, Thomas MR, Shanafelt TD. Systematic review of depression, anxiety, and other indicators of psychological distress among U.S. and Canadian Medical students. Acad. med. 2006;81(4):354-73.

28. Lima M, Soares BGO, Mari JJ. Saúde e doença mental em Pelotas, RS: dados de um estudo populacional. Rev. psiquiatr. clín. 1999;26:225-35.

29. Lima MCP. Transtornos mentais comuns e uso de álcool na população urbana de Botucatu- SP: um estudo de co-morbidade e utilização de serviços São Paulo [tese]. São Paulo: Universidade de São Paulo; 2004.

30. Facundes VLD, Ludermir AB. Common mental disorders among health care students. Rev. bras. psiquiatr. 2005;27(3):194-200.

31. Lima MCP, Domingues MS, Ramos ATAC. Prevalência e fatores de risco para transtornos mentais comuns entre estudantes de medicina. Rev Saúde Públ 2006;40(6)1035-1041.

32. Soares DFG, Moura-Faico MMM. Transtornos mentais em estudantes de medicina. Caratinga: FUNEC; 2010.

33. Zonta R, Robles ACC, Grosseman S. Estratégias de enfrentamento do estresse desenvolvidas por estudantes de medicina. Rev. bras. educ. med. 2006;30(3):147-53.

\section{CONTRIBUICÃO DOS AUTORES}

Railly Crisóstomo Silva participou do delineamento da pesquisa, da coleta de dados, da análise dos dados e da redação do artigo. Alexandre de Araújo Pereira participou do delineamento da pesquisa e da revisão do artigo. Eliane Perlatto Moura participou do delineamento da pesquisa, da coleta de dados, da análise dos dados e da redação do artigo e da revisão do artigo.

\section{CONFLITO DE INTERESSES}

Os autores declaram não haver conflito de interesses neste estudo.

\section{ENDEREÇO PARA CORRESPONDÊNCIA}

Eliane Perlatto Moura. Universidade José do Rosário Vellano, Unifenas-BH, Rua Líbano, 66, Itapoã, Belo Horizonte, MG, Brasil. CEP: 31710-030.

E-mail: elianeperlatto@gmail.com 